\title{
Intervenir en lengua oral y lengua escrita para mejorar las competencias académicas del alumnado de educación secundaria obligatoria
}

\author{
Oral and Written Language Interventions to Improve \\ the Academic Competence of High-School Students
}

\author{
Paula Resina \& Naymé Salas
}

Universitat Autònoma de Barcelona, España

\section{Resumen}

\begin{abstract}
La lengua oral y la lengua escrita están en constante relación en comunidades alfabetizadas. En este artículo se aborda cómo repercute la enseńanza del discurso argumentativo oral en el desarrollo del discurso argumentativo escrito, y viceversa. Los participantes fueron alumnos de $1^{\circ}$ curso de educación secundaria, asignados aleatoriamente a una de tres condiciones: el grupo Oral-1 recibió una intervención en lengua oral primero y en lengua escrita después; el grupo Esc-1 recibió una intervención en lengua escrita primero, y en lengua oral después, y un grupo de control, que recibió las clases habituales de Lengua y Literatura Castellana. Se realizaron evaluaciones pretest, postest- 1 y postest- 2 para determinar la mejora en habilidades intramodalidad e intermodalidad. Los resultados indicaron que los efectos intramodalidad de la intervención en lengua escrita fueron más contundentes que los de la intervención en lengua oral. Ambas intervenciones produjeron efectos de intermodalidad. Se concluye que la enseñanza del discurso argumentativo debe tener en cuenta las relaciones entre ambas modalidades.
\end{abstract}

Palabras clave: desarrollo de la lengua escrita, desarrollos tardíos del lenguaje, intervención educativa, SRSD, español

Correspondencia a:

Naymé Salas

Dept de Didàctica de la Llengua i la Literatura, i de les Ciències Socials

Facultat de Ciències de l'Educació, Universitat Autònoma de Barcelona

Campus de Bellaterra, 08193 - Cerdanyola del Vallès, Barcelona, España.

nayme.salas@uab.cat

$+34935811678$

(C) 2021 PEL, http://www.pensamientoeducativo.org - http://www.pel.cl 


\begin{abstract}
Oral and written language are constantly interrelated in literate communities. In this paper, we examine the repercussions of teaching argumentative oral discourse on the development of argumentative writing and vice versa. The participants were seventh-grade students, who were randomly assigned to one of three groups: the Oral-1 group, which received an oral language intervention first, followed by a written language intervention; the Esc-1 group, which received the written language intervention first, followed by the oral intervention, and the control group, who attended regular Spanish Language and Literature classes. We assessed measures of both oral and written argumentative discourse at pretest, posttest-1, and posttest- 2 to determine the development of both intramodality and intermodality skills. The results showed that intramodality effects were larger for the written than for the oral language intervention. Both interventions produced intermodality effects. It is concluded that the teaching of argumentative discourse should incorporate the bidirectional influences between the oral and written modalities.
\end{abstract}

Keywords: writing development, later language development, educational intervention, SRSD, Spanish

La lengua oral y la lengua escrita están en constante relación en comunidades alfabetizadas debido a que la lengua oral se ve reflejada en la lengua escrita de múltiples formas y, a su vez, la lengua escrita define gran parte de las representaciones lingüísticas de los hablantes. El concepto de linguistic literacy, propuesto por Ravid y Tolchinsky (2002), recoge la naturaleza de la relación entre ambas modalidades del lenguaje, poniendo de manifiesto que el vínculo entre la lengua oral y la lengua escrita es tan estrecho que es prácticamente imposible comprender el desarrollo de una sin valorar el impacto de la otra. Aunque la lengua oral forma parte del desarrollo cognitivo básico de los seres humanos, muchos de sus usos en situaciones comunicativas específicas se adquieren a lo largo de la escolaridad y más allá, en los llamados desarrollos tardíos del lenguaje. Este término alude a los desarrollos lingüísticos que ocurren a partir de los cinco años, es decir, cuando los aspectos básicos del lenguaje ya han sido adquiridos. Los desarrollos tardíos se caracterizan por añadir nuevas funciones (discursivas) a elementos ya adquiridos (Aparici, 2010; para una descripción detallada del concepto, véase Tolchinsky, 2004).

Por su parte, la lengua escrita y su dominio resultan imprescindibles para garantizar el éxito académico, profesional y personal. Por ello, la enseñanza eficaz de la lengua oral y de la lengua escrita son un desafío educativo clave, el cual debe tener en cuenta la relación y posibles sinergias entre ambas modalidades de producción lingüística. Este artículo reporta una investigación sobre la relación entre la lengua oral y la lengua escrita en el contexto específico del desarrollo de la argumentación, entendida como un género discursivo, esto es, textos (orales o escritos) que responden a expectativas culturales específicas sobre su forma y función, y que presentan una articulación particular de características lingüísticas asociadas con dicho género (Biber, 2010; Biber \& Conrad, 2009). Específicamente, cómo repercute la enseñanza del discurso argumentativo oral en el desarrollo del discurso argumentativo escrito y viceversa: cómo repercute la enseñanza del discurso argumentativo escrito en el desarrollo del discurso argumentativo oral. 


\section{Relación entre lengua oral y lengua escrita}

La lengua oral y la escrita se caracterizan por su complementariedad e influencia recíproca (Nelson, 2010; Ravid \& Tolchinsky, 2002). En ambas modalidades se utilizan conocimientos similares para cada componente del lenguaje; por ejemplo, se aplica conocimiento fonológico para entender y producir el habla, así como para la decodificación durante la lectura, o para la ortografía, en la escritura (Caravolas et al., 2012; Gaskell \& MarslenWilson, 1998). Además, tanto el lenguaje oral como el escrito requieren representaciones morfosintácticas (Levesque, Kieffer, \& Deacon, 2017; Treiman, 2017) y discursivas (Kintsch \& Van Dijk, 1978; Wen \& Coker, 2020) para formular los mensajes. No sorprende, pues, que estudios previos hayan reportado que la lengua oral predice la expresión escrita (Dockrell \& Connelly, 2015; Spencer \& Petersen, 2018) y, de manera similar, que ciertos aspectos de la lengua escrita favorecen el desarrollo de la lengua oral (Hulme, Zhou, Tong, Lervåg, \& Burgoyne, 2019; Martins \& Silva, 2006; Perfetti, Beck, Bell, \& Hugues, 1987; Vernon \& Ferreiro, 1999).

Sin embargo, el grado de transferencia entre modalidades (oral y escrita) depende del género o subgénero discursivo (Vázquez, 2014). Cada género discursivo se define a partir de un uso específico del repertorio lingüístico; es decir, existen aspectos discursivos que dependen de la modalidad en la que ocurren. Por ejemplo, dentro de un mismo género (por ejemplo, narración, exposición), los textos escritos suelen presentar vocabulario más sofisticado y mayor complejidad sintáctica que los textos orales (Berman \& Nir, 2010; Ravid \& Berman, 2010). Por un lado, esto se debe a que la producción escrita permite volver sobre lo escrito; así, una oración compleja puede ser releída tantas veces como se desee. Al mismo tiempo, la interacción en diferido entre escritor y lector propia del medio escrito requiere de mayor precisión en la expresión, lo cual se consigue con un vocabulario más riguroso y con estructuras sintácticas complejas (Ravid \& Berman, 2010; Salas, 2010). Al mismo tiempo, se ha sugerido que un mismo género (por ejemplo, argumentación) sigue patrones de desarrollo similares en ambas modalidades (orales y escritas). Esto podría deberse a que, al argumentar, ambas modalidades tienen como objetivo principal el uso de técnicas discursivas destinadas a provocar la adhesión de las personas a una tesis a partir de argumentos (Perelman,1997).

En resumen, el desarrollo del discurso en un mismo género, como el argumentativo, en las modalidades oral y escrita presenta aspectos comunes y divergencias. Resulta de interés educativo, por tanto, identificar el potencial de mejorar una de las modalidades (por ejemplo, escrita) a partir de una intervención en la otra modalidad (por ejemplo, oral). La investigación que se abordó en este estudio podría tener, por tanto, importantes implicaciones tanto prácticas (i.e., para la didáctica del lenguaje oral y escrito) como teóricas (i.e., las relaciones bidireccionales entre la lengua oral y la lengua escrita).

\section{Enseñanza del lenguaje oral y escrito}

En el sistema educativo español se examinan diversas situaciones comunicativas con el objetivo de promover la competencia de los alumnos en usos del lenguaje bien demarcados socialmente (Decreto 187/2015). De los diversos géneros discursivos que se trabajan en clase, el tratamiento del discurso argumentativo es una prioridad debido a (1) la importancia de la argumentación para la construcción y regulación del pensamiento (Larraín, Freire, \& Olivos, 2014); (2) la dificultad inherente al género en cuestión (Coirier \& Golder, 1993), y (3) que las habilidades de argumentación se continúan desarrollando hasta edades avanzadas (Felton \& Kuhn, 2001; Golder \& Coirier, 1996; Scholtz, Sadeck, Hodges, Lubben, \& Braund, 2006). 


\section{Enseñanza del discurso argumentativo oral}

El desarrollo de la argumentación oral se extiende más allá de la adolescencia (Felton \& Kuhn, 2001). La enseñanza de la argumentación en el aula de secundaria debe proporcionar a los alumnos modelos y técnicas para estructurar sus argumentos y actuar de manera estratégica (Dickinson \& Tabors, 2001; Reznitskaya, Anderson, \& Kuo, 2007; Telenius, Yli-Panula, Vesterinen, \& Vauras, 2020). Un tipo de intervención pedagógica, el programa Word Generation (WG), ha mostrado ser efectivo para mejorar el rendimiento en argumentación oral. Se basa en promover discusiones en el aula, explicitando cómo elaborar argumentos coherentes y efectivos y haciendo hincapié en usos académicos del lenguaje (Duhaylongsod, 2017; Jones et al., 2019).

WG se caracteriza por introducir temas controvertidos de discusión junto a actividades estructuradas. Así, proporciona modelos de discurso argumentativo oral y estrategias de discusión (Jones et al., 2019). Por otra parte, el lenguaje académico hace referencia a aquellos recursos lingüísticos de alta frecuencia en textos académicos de distintas disciplinas que tienden a ocurrir con poca frecuencia en el lenguaje coloquial (Uccelli \& Meneses, 2015). Por ejemplo, en el lenguaje académico se utiliza una cantidad más elevada de palabras con morfología derivada (Kieffer \& Lesaux, 2010), conectores más formales (por ejemplo, en otras palabras, eventualmente, ciertamente; Hyland, 2004), oraciones con estructuras gramaticales más complejas que las que tienden a aparecer en el lenguaje coloquial (Taylor, Greenberg, Laures-Gore, \& Wise, 2011), entre otros recursos lingüísticos (para una descripción detallada de las habilidades clave del LA, véase Uccelli et al., 2015 y Meneses et al., 2018).

La combinación de discusiones en el aula con la enseñanza del lenguaje académico del programa WG mejora la calidad discursiva; es decir, la capacidad de posicionarse respecto de una tesis a partir de argumentos, junto con la capacidad de rebatir o apoyar los argumentos de otros (Duhaylongsod, 2017). En este estudio, se aplicó una intervención didáctica basada en el modelo de WG (SERP, s.f.).

\section{Enseñanza de la argumentación escrita}

La construcción de textos escritos es una tarea compleja que requiere la orquestación de numerosos procesos y niveles de representación lingüística (Alamargot \& Chanquoy, 2001). La enseńanza de la escritura, por lo tanto, debe centrarse en proporcionar estrategias explícitas de los diferentes procesos de composición escrita, así como movilizar conocimiento discursivo y lingüístico. El modelo Self-regulated Strategy Development (SRSD) para enseñar a escribir consiste en facilitar el conocimiento de las características discursivas de los textos (por ejemplo, la estructura de los textos), explicitando procesos de escritura (como la planificación o la revisión) y promoviendo el uso de estrategias de autorregulación (por ejemplo, auto instrucciones, autoevaluación), las cuales ayudan a los estudiantes a gestionar los diferentes procesos de escritura. El docente de las intervenciones SRSD suele enfatizar actitudes positivas hacia la escritura y proporciona un modelo de uso de las estrategias de escritura y de autorregulación. Además, orienta al alumno en su camino hacia el uso autónomo de las estrategias enseñadas (Graham \& Harris, 2018). La eficacia del SRSD para la enseñanza de la escritura ha sido comprobada en numerosos estudios y metaanálisis (Salas, Birello \& Ribas, 2020; Graham, McKewon, Kiuhara, \& Harris, 2012; Graham $\&$ Perin, 2007). Este estudio aplicó una intervención SRSD para enseñar a planificar textos argumentativos.

\section{El estudio}

El objetivo principal del estudio fue explorar la relación entre la lengua oral y la escrita. Específicamente, se investigó la influencia del desarrollo de la argumentación oral en el de la argumentación escrita y viceversa. Para ello, se adoptó una metodología cuasiexperimental, en la que se comparó a tres grupos de alumnos, que fueron asignados aleatoriamente a una de tres condiciones: Oral-1, grupo que recibió una intervención en lengua oral primero y en lengua escrita después; Esc-1, que recibió una intervención en lengua escrita primero y en lengua 
oral después. Por último, se incluyó un grupo-clase de control, que recibió las clases habituales de Lengua y Literatura Castellana del currículo de primer curso de la ESO (Decreto 187/2015). Se realizaron evaluaciones en tres ocasiones: pretest, postest-1 (al concluir la primera fase de intervención) y postest-2 (al concluir la segunda fase). Este diseño permitió comprobar la efectividad de cada intervención en el rendimiento intramodalidad, comprobando, por ejemplo, la efectividad de la intervención en lengua escrita en el grupo Esc-1 al final de la primera fase (postest-1); es decir, cuando acaba de recibir la intervención en lengua escrita. Además, el diseño permite comprobar las influencias intermodalidad, comprobando, por ejemplo, el nivel de lengua oral del grupo Esc-1 en postest-1, cuando aún no ha recibido la intervención en lengua oral.

Así, el estudio pretendió responder a dos preguntas de investigación. En primer lugar, ¿¿Son las intervenciones realizadas eficaces para mejorar el rendimiento intramodalidad? Hipotetizamos que la respuesta sería afirmativa, es decir, que la intervención en lengua oral mejorará el rendimiento en lengua oral y la intervención en lengua escrita mejorará el rendimiento en lengua escrita. La hipótesis se asienta en los precedentes de eficacia intramodalidad tanto de la intervención en lengua oral (Venville \& Dawson, 2010) como de la intervención en lengua escrita (Salas, Birello \& Ribas, 2020; Graham et al., 2012). En segundo lugar, nos preguntamos: ¿Existe evidencia de efectos intermodalidad? En este caso, hipotetizamos que la respuesta sería también afirmativa; es decir, la intervención en lengua oral producirá mejoras en el rendimiento en lengua escrita y viceversa: la intervención en lengua escrita producirá mejoras en el rendimiento en lengua oral. Esta hipótesis se basa en el concepto de linguistic literacy; es decir, en el hecho de que la lengua oral y escrita se influyen mutuamente a lo largo del desarrollo en comunidades alfabetizadas (Ravid \& Tolchinsky, 2002).

\section{Método}

\section{Participantes}

Los participantes fueron 50 alumnos $(M$ edad $=12,3$; D.E. $=0,77)$, de $1^{\circ}$ curso de Educación Secundaria Obligatoria (ESO), es decir, estudiantes en plena transición de la educación primaria a la secundaria. Procedían de un instituto público de la provincia de Barcelona, España, región en la que la lengua en la que se imparten las materias es el catalán. Los alumnos reciben, además, clases de Lengua Castellana en la misma proporción que el resto del estado (Real Decreto 1105/2014). Los alumnos estaban organizados en tres grupos-aula. De acuerdo con el comité de ética de la universidad, los tutores legales de los estudiantes y los propios estudiantes firmaron formularios de consentimiento informado para participar en el estudio.

Debido al particular contexto lingüístico de los participantes, estos fueron invitados a completar un cuestionario sociolingüístico. El 94,23\% de la muestra había realizado toda su escolarización obligatoria (desde los seis años) en Cataluña, mientras que solo 5,77\% había empezado su escolarización en Cataluña posteriormente. Las características lingüísticas de la muestra reflejan que, en situaciones familiares, $75 \%$ de los estudiantes hablaba castellano con sus padres/tutores legales, 11,54\% hablaba catalán y el 13,46\% restante hablaba otras lenguas. Con los hermanos, 75,47\% hablaba castellano, 7,69\% hablaba catalán, y 3,85\% hablaba otras lenguas. En las relaciones sociales, $100 \%$ de la muestra usaba el castellano como lengua principal entre amigos y compañeros. Estos datos indican que la muestra del estudio era, fundamentalmente, castellanohablante. En relación con el nivel de estudios de los progenitores, $16,34 \%$ tenía estudios universitarios completados, $64,42 \%$ estudios secundarios obligatorios o de formación profesional, y el 20,19\% restante solo tenía estudios primarios completos.

Los estudiantes que participaron fueron divididos en tres grupos: un grupo experimental Oral-1, formado por 16 estudiantes (nueve niñas) que recibieron una intervención en la modalidad oral seguida de una intervención en la modalidad escrita; otro grupo experimental Esc-1, formado por 17 estudiantes (nueve niñas), que recibieron 
una intervención en la modalidad escrita seguida de la intervención en la modalidad oral, y un grupo de control, integrado por 17 alumnos (siete niñas), que recibió las clases habituales de la asignatura Lengua y Literatura Castellana. La asignación de los participantes a cada grupo se realizó de manera aleatoria, manteniendo a cada alumno en su grupo-clase; es decir, se asignó cada grupo-clase a una condición.

\section{Las intervenciones}

Las intervenciones educativas se realizaron durante el primer semestre del curso escolar, en sesiones que tenían lugar una o dos veces a la semana, como sustituto parcial del temario de la asignatura Lengua Castellana y Literatura. Las intervenciones se realizaron de manera simultánea en los dos grupos experimentales para asegurar la comparabilidad y fueron impartidas por la primera autora. Las intervenciones tenían una duración de ocho sesiones de una hora. Así, la duración total en ambas modalidades era de 16 sesiones u horas, separadas en el medio por tres semanas antes de realizar el cambio de condición (por ejemplo, de oral a escrito).

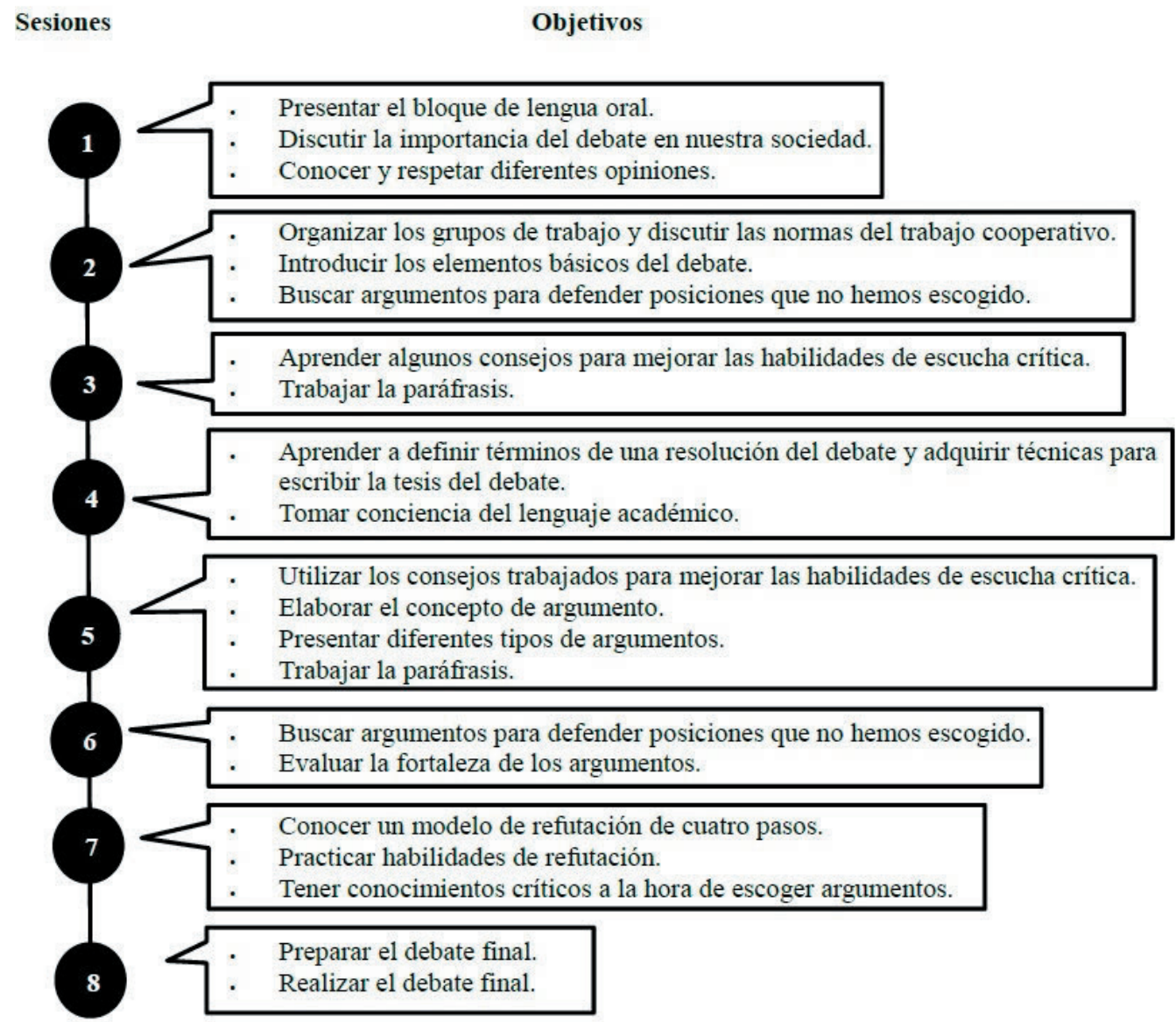

Figura 1. Detalle intervención en lengua oral

Fuente: Elaboración propia. 
Para la intervención en lengua oral (figura 1), se adaptó parte del material del programa Word Generation para estudiantes de la escuela secundaria. Específicamente, se seleccionaron seis actividades del programa destinadas a desarrollar la discusión y el debate académico en el aula. En la primera sesión se explicó a los alumnos la importancia de desarrollar la capacidad de oratoria, exponer ideas en público y discutir sobre temas diversos respetando otras opiniones. En esta primera sesión se trabajó con el material Opinion Continuum $@$, con la finalidad de que los alumnos fueran capaces de asimilar diferentes posturas sobre un mismo tema. En la segunda sesión se adaptó el material Opposite Perspectives@ sobre cómo defender posiciones que no comparten. En la tercera sesión se incidió en la importancia del lenguaje verbal y la escucha a partir del material adaptado de Bonomo, Mamberti y Miller (2010). En la cuarta sesión se dieron pautas para proponer tesis de debate usando lenguaje académico con el material Taking it $U_{p}$ a Level $@$. En la siguiente sesión se siguieron realizando actividades para enseñar a construir argumentos con los que defender el punto de vista propio y el opuesto. Para realizar esta actividad se utilizó una adaptación del material Pro and Con@ $@$. En las sesiones seis y siete se continuó trabajando la argumentación aprendiendo a valorar y diferenciar la calidad de los argumentos. Se utilizó el material Rating Reasons $(\mathrm{y}$ yeighing the Evidence $\bigcirc$. En la sesión ocho, los alumnos realizaron un debate final para poner en práctica las estrategias aprendidas. Asimismo, se utilizaron recursos del libro de Bonomo y sus colegas (2010). Estos se utilizaron para enseñar a los alumnos las características y la estructura del debate mediante contenido teórico.

Todas las sesiones de la intervención en lengua oral presentaron la misma estructura. En cada una de las ocho sesiones se trabajó explícitamente un conjunto de piezas léxicas y expresiones para argumentar en lenguaje académico (figura 2). Al inicio de cada sesión se hizo la lectura de un texto seleccionado previamente, junto con el vocabulario que se trabajaría durante la sesión. Seguidamente, se realizó una actividad en la que se les invitaba a reflexionar y a trabajar diferentes estrategias para ser buenos oradores: refutación, escucha activa, paráfrasis. Cada sesión finalizó con una discusión para practicar los conceptos enseñados.

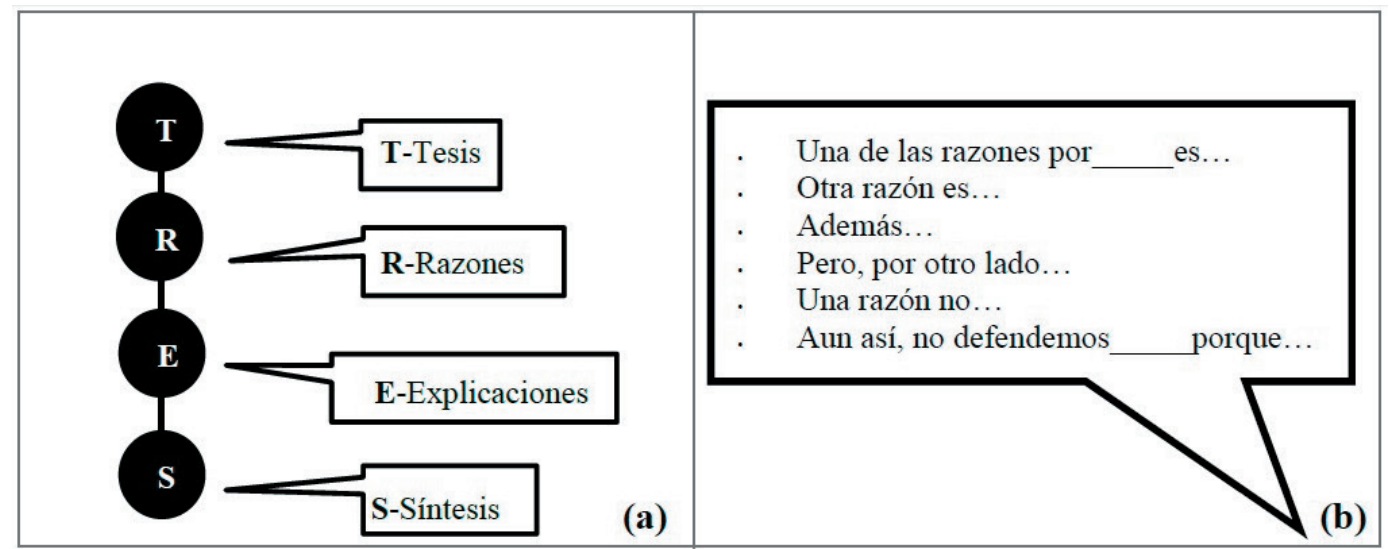

Figura 2. Ejemplo de recursos de las intervenciones. En (a) se muestra la estrategia para planificar textos escritos argumentativos. En (b) se muestra un ejemplo de un conjunto de piezas léxicas y expresiones para debatir usando lenguaje académico.

Fuente: Elaboración propia.

Para la intervención en lengua escrita (figura 3), se adaptó al castellano la intervención utilizada por Salas, Birello \& Cros (en preparación), basada en el modelo SRSD de Graham y Harris (2009), y destinada a la enseñanza de estrategias de planificación de textos argumentativos y de autorregulación. La intervención dedicó las primeras tres sesiones a movilizar conocimientos previos, introducir terminología y presentar la estrategia de escritura (figura 2) y las estrategias de autorregulación. En la sesión cuatro, la primera autora modeló el proceso 
de escritura utilizando las estrategias presentadas. En las sesiones cinco y seis, los estudiantes practicaron las estrategias con la ayuda de un organizador gráfico, mientras que a partir de la sesión siete se propuso a los estudiantes escribir sin el soporte del organizador gráfico. En definitiva, la intervención pasó progresivamente de la modelización explícita de las estrategias de escritura y autorregulación por parte del docente hacia el uso autónomo de estas por parte del alumnado.

Los estudiantes de todos los grupos completaron, además, una prueba pretest una semana antes del inicio de la primera fase de la intervención, una prueba postest-1, una semana después de la primera fase de intervención, y una prueba postest-2, una semana después de la segunda fase de intervención.

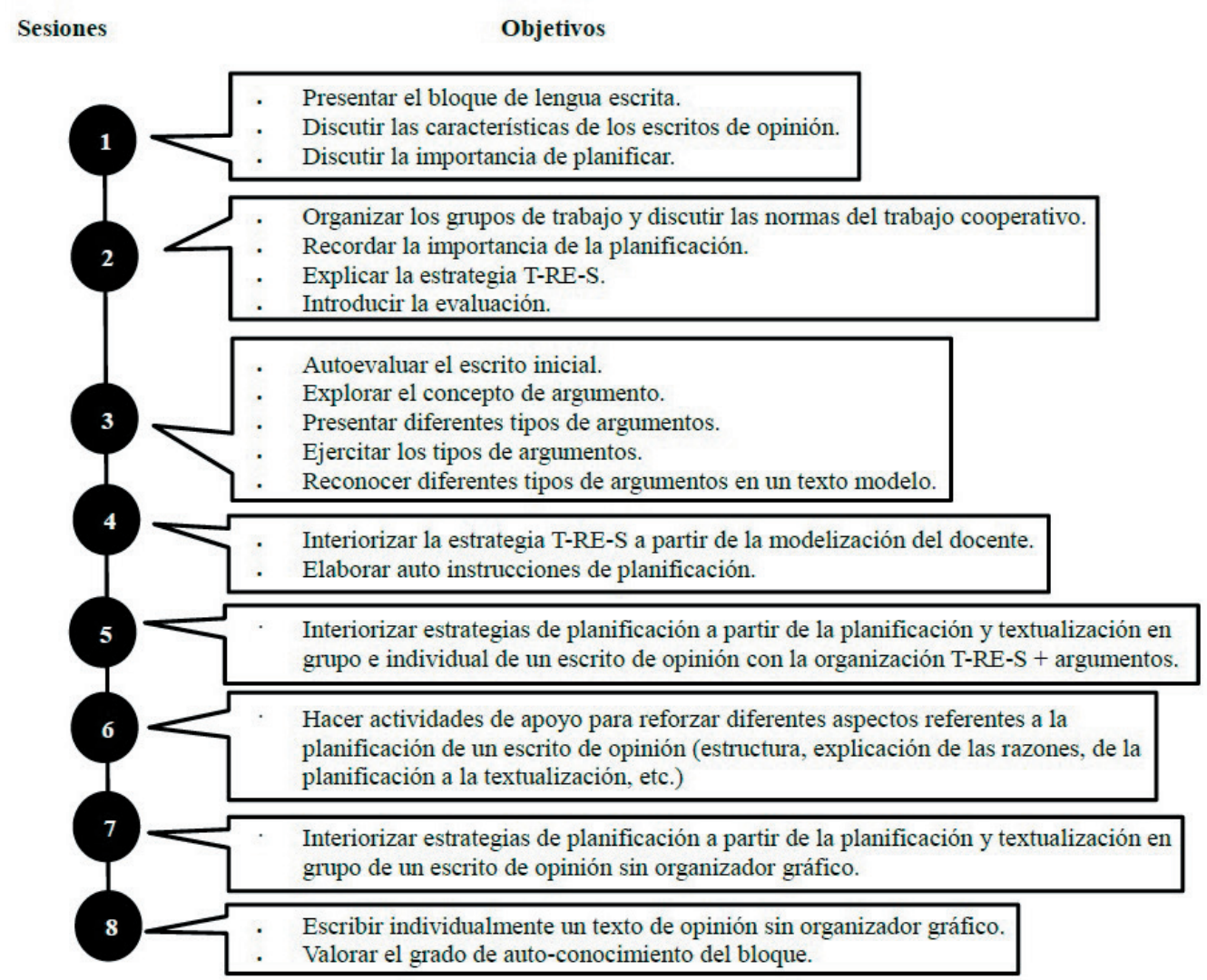

Figura 3. Detalle intervención en lengua escrita

Fuente: Elaboración propia.

\section{Tareas de lengua oral y lengua escrita}

\section{Argumentación oral}

Los participantes, en grupos de cinco alumnos, realizaron una breve discusión de cinco minutos a partir de un texto leído por la primera autora. En el pretest, el texto trataba del trabajo infantil; en el postest-1, de la jornada escolar intensiva, y en el postest-2, del uso de Internet por parte de los adolescentes. 


\section{Lenguaje académico}

Para determinar el nivel de lenguaje académico se utilizó el Instrumento de Evaluación de Lenguaje Académico (ELA), destinado a alumnos hispanohablantes de $4^{\circ}$ a $8^{\circ}$ grado (Meneses et al., 2018). El instrumento incluye nueve tareas que evalúan conocimiento del lenguaje a nivel léxico, gramatical y discursivo de recursos propios del lenguaje académico. Nótese que, aunque se trata de una prueba que se realiza por escrito, refleja conocimientos aplicables al género argumentativo que abarcan usos tanto orales como escritos. Dado que la intervención en lengua oral trabajaba explícitamente el uso del lenguaje académico en el contexto de la discusión, se la ha considerado, primordialmente, una medida de lengua oral en este estudio.

\section{Argumentación escrita}

Los participantes escribieron un texto de opinión durante un máximo de 15 minutos. Previamente, tenían hasta ocho minutos para planificar el texto en una hoja borrador. En el pretest, la consigna de escritura fue ¿Crees que todas las personas de tu edad tienen que hacer la ESO?; en el postest-1, la consigna de escritura fue ¿Crees que es necesario alargar el tiempo del recreo?, y en el postest-2 la consigna de escritura fue ¿Crees que en el instituto es mejor el uso de portátiles que el de libros de texto? Los estudiantes no recibieron ningún tipo de ayuda para resolver problemas de contenido o estructura, como tampoco de estilo u ortografía.

\section{Medidas}

\section{Calidad del discurso oral}

Los debates fueron transcritos y se evaluaron utilizando una adaptación de la Discussion Rubric@ del programa Word Generation (WG; SERP, s.f). La rúbrica presentaba un total de seis dimensiones: la exteriorización del punto de vista del alumno, el uso de la evidencia del texto, el uso de las ideas de los otros, el uso del lenguaje académico, el estilo de los argumentos y el compromiso de discusión. Cada dimensión recibió una puntuación de 1 a 4, en donde la puntuación más baja (1) correspondía a una valoración de inefectiva y la puntuación más alta (4), a una valoración de efectiva. La nota final resultaba de la suma de las puntuaciones para cada dimensión. La primera autora evaluó todas las transcripciones, mientras que una técnica de soporte a la investigación evaluó $20 \%$ de las transcripciones, escogidas al azar. La fiabilidad entre evaluadoras (ICC) fue ,989.

\section{Lenguaje académico}

Las tareas del ELA están compuestas por ítems de opción múltiple o de respuesta forzada. Para dar el mismo peso a cada una de las tareas, se calculó la proporción de respuestas correctas en cada tarea; así, la puntuación máxima en cada una oscilaba entre 0 y 1 , mientras que la puntuación final del test oscilaba entre 0 y 9 . La primera autora evaluó todos los protocolos y una asistente a la investigación evaluó las respuestas de $20 \%$ de los instrumentos escogidos al azar. La fiabilidad (ICC) fue, 996.

\section{Calidad del texto escrito}

Los textos escritos se transcribieron de manera literal y luego fueron evaluados para comprobar hasta qué punto incluían elementos estructurales propios del discurso argumentativo (siguiendo a Salas, Birello \& Ribas, 2020; Coirier \& Golder, 1993; Limpo \& Alves, 2013; Wagner et al., 2011). Específicamente, se otorgó un punto por 
la presencia de una introducción que diera contexto y un punto por expresar la tesis u opinión explícitamente. Se otorgaba, además, un punto si el texto presentaba una conclusión. También se otorgaba un punto por cada razón proporcionada, así como por cada elaboración o explicación de las razones. Solo se otorgaron puntos por elementos claramente observados y no se otorgaron puntuaciones parciales (por ejemplo, por razones incompletas). La puntuación final resultaba de la suma de todos los elementos incluidos por el estudiante en el texto. La primera autora evaluó todos los escritos y una asistente a la investigación, ajena al propósito del estudio, evaluó $20 \%$ de los textos escogidos al azar. La fiabilidad (ICC) fue ,992.

\section{Longitud del texto}

El número total de palabras fue utilizado para estimar la longitud del texto; es decir, el nivel de productividad en lengua escrita, que suele ser un índice cercano a la calidad global que se establece de manera temprana en diferentes lenguas (Salas \& Caravolas, 2019; Berman \& Verhoeven, 2002; Berman \& Nir, 2010; Malvern, Richards, Chipere, \& Durán 2004). Los errores de ortografía no fueron considerados para el recuento de palabras; por lo tanto, si una palabra contenía un error de ortografía se sumaba de igual manera que si estaba escrita convencionalmente. Las creaciones léxicas o cambios puntuales de código a la otra lengua que hablan los estudiantes (catalán) también se incluyeron en el recuento total.

\section{Procedimiento}

Las evaluaciones del pretest, postest-1 y postest- 2 fueron llevadas a cabo por la primera autora. La prueba de calidad del discurso oral tuvo lugar en una habitación silenciosa dentro del recinto escolar. El resto de las pruebas fue administrado en el aula habitual de clase. Las diferentes evaluaciones se realizaron la tercera semana del mes de octubre (pretest), la tercera semana de diciembre (postest-1) y la tercera semana de febrero (postest-2), siempre respetando la hora asignada a la asignatura Lengua Castellana y Literatura y sin administrar más de una prueba diaria por grupo. El orden de las pruebas fue el mismo en todos los grupos: lengua oral, lengua escrita, y lenguaje académico.

\section{Plan de análisis}

Para cada una de las medidas de lengua oral y escrita se realizaron análisis de varianza (ANOVA, según las siglas en inglés) mixtos de dos factores: tiempo, variable intrasujeto con tres valores: pretest, postest-1, postest-2, y grupo, variable intersujeto con tres valores: Oral-1, Esc-1 y control. Para el análisis del factor tiempo se realizaron comparaciones planeadas (método repeated en el programa SPSS v. 20) de los intervalos pretest>postest-1 y postest-1>postest-2. Para el análisis del factor grupo se realizaron análisis post-hoc Bonferroni, para determinar si la pertenencia a alguno de los grupos afectaba el rendimiento en cada una de las habilidades observadas. Por último, en el seguimiento de las interacciones significativas se utilizó la corrección Bonferroni.

\section{Resultados}

La tabla 1 muestra los resultados descriptivos de las evaluaciones pretest, postest- 1 y postest- 2 de las dos habilidades observadas de lengua oral: calidad del discurso oral y lenguaje académico, y las dos habilidades observadas de lengua escrita: calidad del texto escrito y longitud del texto. Todas las variables presentaban una distribución normal, con una asimetría máxima de $\pm 0,99$ y una curtosis máxima de $\pm 1,4$ (Kline, 2011). 


\section{Impacto de las intervenciones en el aprendizaje de la lengua oral}

El efecto tiempo tuvo un fuerte impacto en la calidad discursiva oral, la cual experimentó una mejora significativa, $F(2,90)=89,57 ; p<, 001 ; \eta_{\mathrm{p}}^{2}=0,666$. Dicha mejora fue observada tanto en el intervalo del pretest al postest-1 $(p<, 001)$, como del postest-1 al postest-2 $(p<, 001)$. Sin embargo, no todos los grupos mejoraron su rendimiento en calidad discursiva oral en la misma medida, dado que hubo un efecto comparativamente más pequeño, pero significativo de grupo, $F(2,45)=5,39 ; p=, 008 ; \eta_{\mathrm{p}}^{2}=0,193$. Los análisis post-hoc indicaron que el grupo Oral-1 experimentó una mejora significativamente superior al grupo control $(p=, 032)$ y al grupo Esc-1 $(p=, 012)$, mientras que los grupos Esc-1 y control experimentaron mejoras similares $(p>, 05)$. La interacción no fue significativa, $F(4,90)=2,28 ; p=, 067 ; \eta_{\mathrm{p}}^{2}=0,092$.

Tabla 1

Estadística descriptiva de las medidas de lengua oral y lengua escrita de cada grupo y tiempo de recogida.

$\begin{array}{ccc}\text { Oral-1 } & \text { Esc-1 } & \text { Control } \\ M(\mathrm{DE}) & M(\mathrm{DE}) & M(\mathrm{DE})\end{array}$

\section{Pretest}

\begin{tabular}{cccc}
\hline Calidad discurso oral & $11,50(5,24)$ & $9,47(2,57)$ & $11,44(2,38)$ \\
\hline Calidad texto escrito & $4,13(1,20)$ & $3,47(1,62)$ & $3,82(0,88)$ \\
\hline Longitud del texto & $86,50(28,74)$ & $64,76(30,09)$ & $77,71(29,92)$ \\
\hline Lenguaje académico & $5,23(1,98)$ & $5,71(1,46)$ & $5,37(1,39)$ \\
\hline
\end{tabular}

Postest-1

\begin{tabular}{cccc}
\hline Calidad discurso oral & $18,33(4,35)$ & $13,88(4,24)$ & $13,88(3,99)$ \\
\hline Calidad texto escrito & $5,80(2,45)$ & $8,59(2,26)$ & $3,47(1,06)$ \\
\hline Longitud del texto & $98,93(48,88)$ & $119,88(32,01)$ & $59,18(18,53)$ \\
\hline Lenguaje académico & $6,96(0,98)$ & $5,81(1,30)$ & $5,39(1,40)$ \\
\hline
\end{tabular}

Postest-2

\begin{tabular}{cccc}
\hline Calidad discurso oral & $21,27(3,32)$ & $18,65(3,82)$ & $17,69(4,02)$ \\
\hline Calidad texto escrito & $10,73(1,75)$ & $10,38(1,45)$ & $3,65(1,41)$ \\
\hline Longitud del texto & $146,07(44,23)$ & $132,06(28,66)$ & $56,35(26,77)$ \\
\hline Lenguaje académico & $6,88(0,90)$ & $6,34(1,30)$ & $5,99(1,38)$ \\
\hline
\end{tabular}

Fuente: Elaboración propia.

El efecto tiempo tuvo un impacto moderado en el lenguaje académico, $F(2,86)=26,22 ; p<, 001 ; \eta_{\mathrm{p}}^{2}=, 379$, mientras que no hubo un efecto principal de grupo, $F(2,43)=2,29 ; p=, 114 ; \eta_{\mathrm{p}}^{2}=, 096$. Se observó una interacción significativa, comparativamente más pequeña, $F(4,86)=4,82 ; p=, 001 ; \eta_{\mathrm{p}}^{2}=, 183$ (figura 4). El seguimiento de esta interacción indicó que el efecto simple de la variable tiempo fue significativo para el grupo Oral-1, $F(2,26)=6,55 ; p<, 001 ; \eta_{\mathrm{p}}^{2}=, 574$, aunque solo en el intervalo del pretest al postest $1(p<, 001)$. 
El efecto tiempo también fue significativo para el grupo Esc-1, $F(2,30)=2,47 ; p=, 001 ; \eta_{\mathrm{p}}^{2}=, 352$, aunque solo en el intervalo del postest- 1 al postest- $2(p=0,018)$. El efecto simple de tiempo fue solo marginalmente significativo para el grupo control ${ }^{1}, F(1,09 ; 16,41)=6,88 ; p=, 016 ; \eta_{\mathrm{p}}^{2}=, 314$, en donde solo el intervalo del postest-1 al postest-2 fue significativo $(p=, 017)^{2}$. Por otra parte, el efecto simple de grupo solo fue significativo en el postest-1, $F(2,46)=9,49 ; p=, 007$. Los análisis post-hoc determinaron que el grupo Oral-1 fue superior al grupo control $(p=, 006)$, mientras que no se diferenció del grupo Esc-1, a la vez que el grupo control y Esc-1 no se diferenciaron significativamente entre sí ( $p$ s > ,05).

Estos resultados indican que todos los grupos mejoraron su rendimiento en lengua oral, independientemente de haber recibido una intervención en lengua oral o no. El grupo Oral-1, quien recibió la intervención en lengua oral a principios de curso, experimentó una ligera ventaja con respecto del resto de grupos.

\section{Impacto de las intervenciones en el aprendizaje de la lengua escrita}

La calidad de los textos escritos mejoró en las sucesivas evaluaciones, dado que hubo un fuerte efecto principal de tiempo, $F(2,88)=114,19 ; p<, 001 ; \eta_{\mathrm{p}}^{2}=0,722$. Sin embargo, no todos los grupos mejoraron la calidad de los textos escritos en la misma medida, dado que también hubo un efecto de grupo de magnitud similar, $F(2,44)=55,34 ; p<, 001 ; \eta_{\mathrm{p}}^{2}=0,716$. Estos efectos fueron moderados por una importante interacción significativa tiempo x grupo, $F(4,88)=39,47 ; p<, 001 ; \eta_{\mathrm{p}}^{2}=0,642$ (figura 4). El efecto simple de tiempo indicó que el grupo Oral-1 experimentó mejoras significativas en ambos intervalos, del pretest al postest-1 $(p=, 005)$ y del postest-1 al postest-2 ( $p<, 001)$. El grupo Esc-1, por el contrario, solo experimentó una mejora significativa en el primer intervalo $(p<, 001)$, mientras que el grupo control no experimentó ninguna mejora significativa en ningún intervalo $(p s \geq, 37)$.

Por otro lado, el efecto simple de grupo no resultó significativo en el puntaje de calidad del texto escrito del pretest $(p=, 345)$, mientras que sí hubo diferencias significativas entre los grupos en el postest- 1 y el postest- 2 $(p s<, 001)$. En el postest-1 los análisis post hoc revelaron que el grupo Esc-1, que acababa de recibir la intervención en lengua escrita, fue superior al resto de grupos ( $p s<, 001$ ), mientras que el grupo Oral-1 superó en promedio al grupo control $(p=, 006)$. En el postest-2, tanto el grupo Esc-1 como el grupo Oral-1 superaron al grupo control $(p s<, 001)$, aunque no se diferenciaron significativamente entre sí $(p>, 05)$.

En cuanto el efecto de las intervenciones en la longitud del texto, se observó un efecto principal de tiempo que fue significativo, $F(2,88)=23,64 ; p<, 001 ; \eta_{\mathrm{p}}{ }_{\mathrm{p}}=0,349$. Asimismo, se observó un efecto significativo de grupo, $F(2,44)=18,12 ; p<, 001 ; \eta_{\mathrm{p}}^{2}=0,452$. Igual que con la calidad del texto escrito, estos efectos principales fueron moderados por una interacción significativa, $F(4,88)=20,36 ; p<, 001 ; \eta_{\mathrm{p}}^{2}=0,481$ (Figura 4). El seguimiento de la interacción indicó que el efecto simple de tiempo en el primer intervalo, pretest-postest-1, fue significativo para el grupo Esc-1 $(p>, 001)$ y para el grupo de control $(p=, 003)$. No obstante, mientras que el grupo Esc-1 incluyó significativamente más palabras, en promedio, en el postest-1 respecto del pretest, el grupo control incluyó significativamente menos palabras (tabla 1). En el segundo intervalo, solo el grupo Oral-1, el cual acababa de recibir la intervención en lengua escrita, incluyó significativamente más palabras del postest-1 al postest-2 $(p=, 001)$, mientras que los otros dos grupos escribieron textos de longitud similar. Por otra parte, el efecto simple de grupo indicó que, aunque en el pretest todos los grupos escribieron textos de una longitud similar ( $p s>, 05)$, en el postest-1 y en el postest-2 los grupos Esc-1 y Oral-1 superaron significativamente al grupo control ( $p s<, 007)$, aunque no se diferenciaron el uno del otro $(p s>, 05)$.

1. Debido a la corrección Bonferroni para comparaciones múltiples, la significatividad se interpreta en valores de p por debajo de 0,017).

2. Se reportan grados de libertad con la corrección Greenhouse-Geisser, debido a la significatividad del test Mauchly de esfericidad. 
Estos resultados indican que ambos grupos de intervención experimentaron mejoras en expresión escrita. Los participantes del grupo control, por el contrario, no mejoraron ni la calidad ni la cantidad de sus textos de opinión en ningún momento del estudio, pese a haber comenzado con niveles similares a los de los otros dos grupos.

(a)

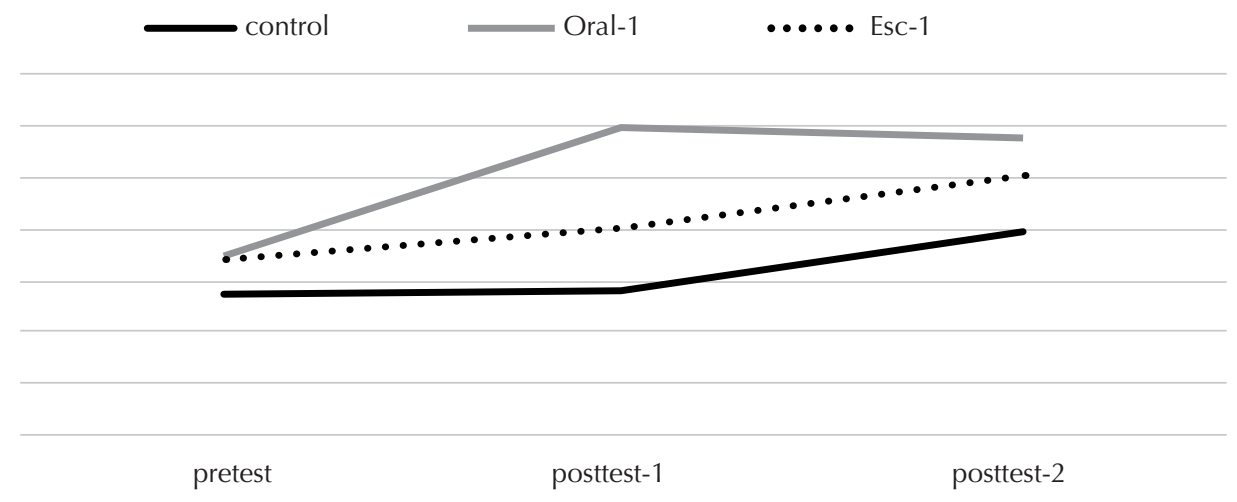

(b)

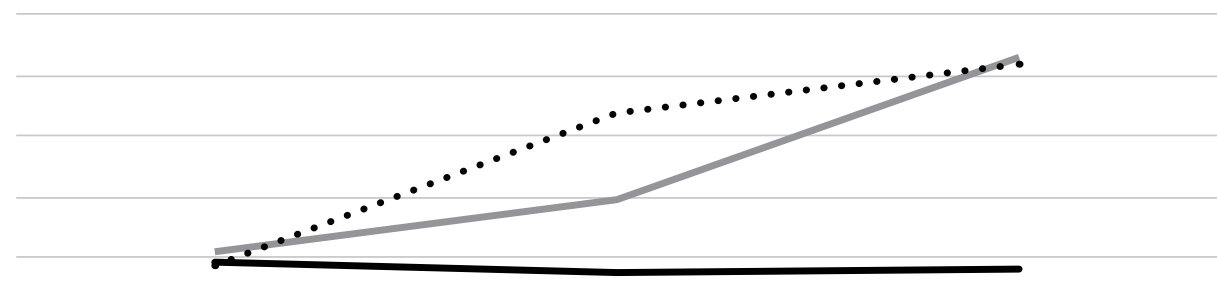

pretest

posttest-1

posttest-2

(c)

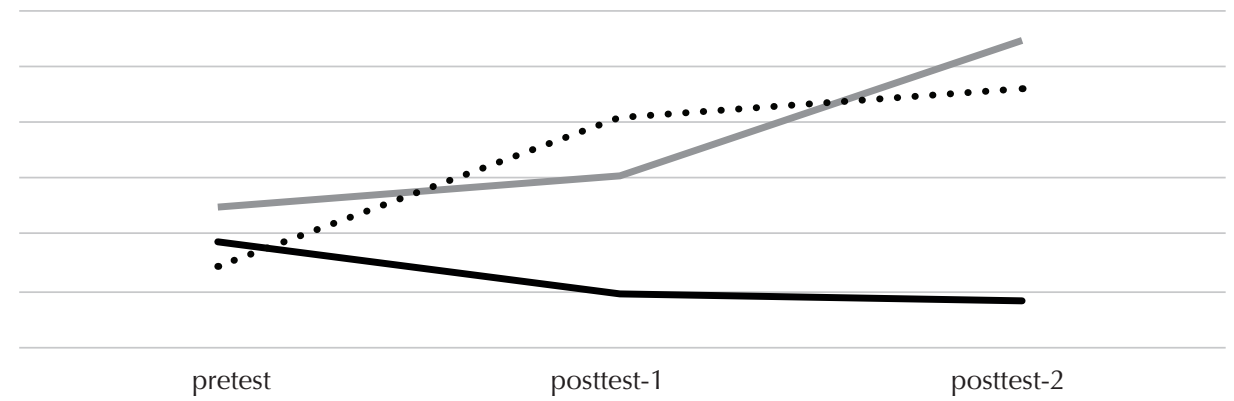

Figura 4. Interacciones significativas entre tiempo y grupo para la variable (a) lenguaje académico, (b) calidad del texto escrito y (c) longitud del texto.(RES)

Fuente: Elaboración propia.

\section{Discusión}

En este estudio nos propusimos investigar las relaciones entre la lengua oral y la lengua escrita en el contexto del desarrollo del discurso argumentativo. Para ello, se implementó una intervención destinada a mejorar el lenguaje argumentativo oral y otra intervención destinada a mejorar el lenguaje argumentativo escrito. 
De esta manera, se examinaron efectos intramodalidad (oral>oral; escrito>escrito) e intermodalidad (oral>escrito; escrito>oral). Estudiantes de primer curso de la ESO fueron divididos en dos grupos: un grupo recibió primero una intervención en lengua oral y luego una intervención en lengua escrita, mientras que otro grupo recibió intervenciones idénticas, pero administradas en orden inverso. Ambos grupos fueron comparados con un grupo de control que recibió las clases habituales de la asignatura de Lengua y Literatura Castellana. Los grupos fueron evaluados antes del inicio de las intervenciones, al finalizar la primera intervención y al finalizar la segunda.

\section{Efectos intramodalidad}

Un primer hallazgo de esta investigación fue que ambas intervenciones mostraron efectos significativos intramodalidad: la intervención en lengua oral mejoró la calidad del discurso argumentativo oral y del lenguaje académico, y la intervención en lengua escrita produjo una mejora en la calidad y la cantidad (longitud del texto en número de palabras) de los textos escritos argumentativos producidos por los participantes. Este hallazgo coincide con investigaciones previas que han demostrado la eficacia del programa Word Generation para la enseñanza de producción de discurso argumentativo oral (Duhaylongsod, 2017) y del programa SRSD para la enseñanza de la expresión escrita (Salas, Birello \& Ribas, 2020; Graham \& Perin, 2007; Graham et al., 2012).

Sin embargo, hubo importantes diferencias en la efectividad de las intervenciones en lengua oral y lengua escrita. La intervención en lengua oral se tradujo en solo una pequeña ventaja para el grupo Oral-1 respecto de los otros dos grupos. Hacia el final del estudio, todos los grupos mostraron niveles de rendimiento en discurso argumentativo oral y lenguaje académico similares. Este hecho requiere interpretaciones diferenciadas para cada uno de los grupos. El grupo Esc-1 mejoró significativamente su rendimiento en lengua oral, seguramente debido a (1) la intervención en lengua oral en el segundo intervalo del estudio y (2) los efectos intermodalidad que se describen en la siguiente sección. La mejora en el rendimiento en discurso argumentativo oral del grupo control, más clara en el segundo período de evaluación, podría indicar que unos meses de educación secundaria son suficientes para observar un incremento similar al de los grupos de intervención en su capacidad para producir un discurso argumentativo oral y utilizar lenguaje académico. Ciertamente, la transición de la primaria a la secundaria en el sistema educativo español se caracteriza por pasar de un único maestro que imparte la mayor parte del temario a la exposición a múltiples docentes, cada uno experto en su materia. Un resultado similar fue reportado en el estudio de Lin, Lawrence, Snow \& Taylor (2016), en donde tampoco se observaron mejoras significativas en la capacidad comunicativa oral del grupo de intervención respecto al grupo control. De la misma manera, en investigaciones previas se ha insistido en que el progreso en discurso argumentativo oral en adolescentes es lento y requiere ańos de intervención educativa para mostrar diferencias significativas con grupos de control (Kuhn \& Crowell, 2011). Por último, resulta sorprendente que los grupos Oral-1 y Esc-1 se diferenciaran entre sí en calidad del discurso argumentativo oral, cuando ambos recibieron la misma intervención, aunque en momentos diferentes. En este sentido, es posible que la intervención más temprana del grupo Oral-1 haya servido para llamar la atención de los participantes hacia ciertos recursos y formas lingüísticas propias de este género discursivo desde el inicio del curso escolar. Así, la movilización de representaciones lingüísticas desde el inicio del curso, sumadas a la exposición a los discursos expertos propios del entorno del aula de secundaria, podrían haber potenciado la efectividad de la intervención en lengua oral en este grupo. Este resultado tiene repercusiones importantes para la planificación de la didáctica de la lengua en secundaria y requiere de investigaciones que corroboren el resultado en muestras más amplias y por un período de seguimiento más extenso.

Al contrario de la intervención en lengua oral, los resultados intramodalidad de la intervención en lengua escrita parecen sugerir que la expresión escrita no progresa sin una enseñanza explícita de los procesos de la escritura. Es decir, la mera exposición a usos más o menos académicos de la lengua escrita propios de la educación secundaria parecen resultar insuficientes para mejorar el rendimiento en escritura. En este sentido, numerosos estudios han 
puesto de manifiesto la importancia de enseñar explícitamente los procesos que intervienen en la escritura, así como de trabajar estrategias de autorregulación que faciliten la ejecución de dichos procesos (Burton, Nunes, \& Evangelou, 2021; Graham \& Harris, 2009; Kemper, Verhoeven \& Bosman 2012).

\section{Efectos intermodalidad}

Tal como se había hipotetizado al inicio del estudio, encontramos evidencia empírica de efectos intermodalidad, en línea con la propuesta de linguistic literacy de Ravid y Tolchinsky (2002). En primer lugar, la intervención en discurso argumentativo oral produjo una mejora en el discurso argumentativo escrito. Al examinar la mejora en calidad del texto escrito del grupo Oral-1 en la primera fase del estudio (pretest a postest-1), cuando el grupo aún no había recibido la intervención en lengua escrita, observamos que superaron al grupo de control. Este resultado indica que el trabajo sobre el debate y la enseñanza explícita del lenguaje académico en la modalidad oral se tradujeron en una ventaja en el momento de producir discurso argumentativo escrito. Dicha ventaja fue además observada tanto en la calidad de los textos escritos, específicamente, en el número de elementos discursivos propios de la argumentación y en su longitud. Estos resultados coinciden con la idea ampliamente sostenida de que el desarrollo de la lengua escrita está soportado, al menos parcialmente, por la lengua oral (Griffin et al., 2004; Juel, Griffith, \& Gough, 1986; Snowling \& Hulme, 2020). En este trabajo, la influencia de una modalidad (oral) sobre otra (escrita) se podría haber visto reforzada por el hecho de que ambas intervenciones trabajaban un mismo género discursivo (argumentación), y a que la intervención oral movilizaba la generación de ideas, así como la calidad y la estructura de los argumentos.

Es importante señalar que se observaron limitaciones al efecto positivo de la lengua oral sobre el aprendizaje de la escritura. Pese a que la intervención en lengua oral contribuyó a una mejora en la expresión escrita, el rendimiento del grupo Oral-1 en la primera fase del estudio fue significativamente inferior al del grupo Esc-1, que había recibido la intervención en lengua escrita. Esto indica, otra vez, que el dominio de la lengua escrita requiere de la enseñanza de estrategias específicas de modalidad. Dicho de otro modo, pese a la evidencia clara de una transferencia de conocimiento de la lengua oral a la escrita, la competencia en escritura requiere, además, la enseńanza explícita de los procesos de escritura y de otros conocimientos propios de la modalidad escrita (Graham \& Perin, 2007).

La intervención en lengua escrita también dio una ventaja al grupo Esc-1 en el rendimiento en lenguaje académico, dado que no mostró diferencias significativas con el grupo Oral-1 en el postest-1 (es decir, cuando el grupo Esc-1 solo había recibido la intervención en lengua escrita). Esto indica que las representaciones discursivas que se movilizan en una intervención de SRSD para escritura argumentativa, movilizan también el repertorio lingüístico propio del registro académico. Ciertamente, existen relaciones forma-función estables entre textos orales y escritos dentro de un mismo género (Berman \& Verhoeven, 2002).

En resumen, la enseñanza explícita de la escritura resulta en un beneficio para el aprendizaje de la modalidad oral y viceversa, en línea con la propuesta de linguistic literacy de Ravid y Tolchinsky (2002) de que el desarrollo del lenguaje en una modalidad no puede entenderse sin referencia a la otra. No obstante, mientras que el tratamiento de la lengua oral propio del entorno escolar de secundaria parece producir un beneficio intramodalidad similar al que se obtiene de una intervención basada en discusiones en el aula y en la promoción del lenguaje académico, una enseñanza de la lengua escrita que proporcione estrategias de escritura y de autorregulación parece imprescindible para obtener mejoras observables en la construcción de discurso argumentativo escrito. Estos resultados implican que, sin detrimento del tratamiento de la lengua oral en el aula de secundaria, el dominio de la lengua escrita requiere que el docente proporcione estrategias explícitas para gestionar la compleja tarea de producir textos argumentativos de calidad. El trabajo de dichas estrategias en el aula deberá ser, probablemente, transversal y sostenido en el tiempo, aunque es necesario investigar más estos matices sobre la enseñanza de la lengua escrita (Graham \& Harris, 2018). 


\section{Implicaciones educativas y observaciones finales}

Una de las principales implicaciones del estudio para la enseńanza del lenguaje argumentativo en el aula de secundaria es que la enseñanza de la lengua oral debe abordarse tan pronto como sea posible, desde el inicio del curso. Dicha enseñanza deberá señalar a los alumnos aspectos relacionados con los usos académicos de la lengua y favorecer las discusiones en el aula, llamando la atención sobre la calidad y estructura de los argumentos. De esta manera, los alumnos aprovecharán más y mejor las oportunidades para adquirir y utilizar el repertorio propio de textos argumentativos en las diferentes disciplinas.

Otra implicación educativa de relevancia tiene que ver con la enseñanza de la escritura. Los resultados de este estudio señalan de manera contundente que la enseñanza de la expresión escrita debe ser explícita y debe proporcionar estrategias y facilitaciones procedimentales.

En conclusión, los hallazgos del estudio se ańaden a la evidencia acumulada hasta el momento de que la lengua oral y la lengua escrita interactúan a lo largo del desarrollo de lenguaje (Berninger 2000; Berninger \& Abbott, 2010; Davidson, 2019; Shanahan, MacArthur, Graham, \& Fitzgerald, 2006), especialmente durante la etapa de los desarrollos tardíos (Tolchinsky, 2004). Por lo tanto, la investigación en adquisición del lenguaje en esta etapa debería atender a las implicaciones de las relaciones bidireccionales entre ambas modalidades, sin dejar de advertir y explicar las especificidades en el aprendizaje de cada modalidad discursiva.

\section{Limitaciones}

Una clara limitación del estudio ha sido el tamaño de su muestra. Una vez que se divide la muestra total en tres grupos, algunos contrastes con una clara tendencia no arrojaron resultados significativos. Futuros estudios deberían obtener muestras más amplias que garanticen el poder estadístico necesario para poder responder de manera más concluyente a las preguntas de investigación planteadas en este trabajo. En segundo lugar, dado que algunas habilidades (por ejemplo, calidad del discurso argumentativo oral) podrían requerir de intervenciones más extendidas en el tiempo para mostrar resultados, una intervención más larga con un seguimiento longitudinal de los diferentes grupos serviría para determinar con mayor solidez el alcance de los efectos de las intervenciones. Por último, los participantes de este estudio tienen un perfil lingüístico particular: hablantes bilingües castellanocatalán, con una clara dominancia del castellano, pero en un entorno en el cual la lengua escolar es el catalán. Es posible que los hallazgos que aquí se reportan no sean generalizables a otros contextos.

Agradecimientos. Esta investigación fue llevada a cabo con el soporte parcial del proyecto Ara, l'escriptura! Millorar l'expressió escrita per garantir l'equitat de l'alumnat (ref. 2015ACUP 00175; I.P. Naymé Salas). Las autoras agradecen inmensamente al personal docente y a los alumnos de $1^{\circ}$ del curso 2018-2019 del IES Santa Eulàlia, de l'Hospitalet de Llobregat de Barcelona. Asimismo, nuestro agradecimiento se extiende a Alondra Camus y a Lucía Redondo por su ayuda en la codificación de una parte de los datos.

El artículo original fue recibido el 15 de enero de 2021

El artículo revisado fue recibido el 17 de julio de 2021

El artículo fue aceptado el 22 de julio de 2021 


\section{Referencias}

Alamargot, D. \& Chanquoy, L. (2001). General Introduction. En D. Alamargot \& L. Chanquoy, Through the models of writing: with commentaries by Ronald T. Kellogg \& John R. Hayes (pp. 1-32). Dordrecht, Holanda: Kluwer Academic Publishers.

Aparici, M. (2010). El desarrollo de la conectividad discursiva en diferentes géneros y modalidades de producción (Tesis doctoral inédita). Universidad de Barcelona, Barcelona, España.

Berman, R. A. \& Nir, B. (2010). The lexicon in writing-speech-differentiation. Written Language \& Literacy, 13(2), 183-205. https://doi.org/10.1075/wll.13.2.01ber

Berman, R. A. \& Verhoeven, L. (2002). Cross-linguistic perspectives on the development of text-production abilities: Speech and writing. Written Language and Literacy, 5(1), 1- 43. https://doi.org/10.1075/wll.5.1.02ber

Berninger, V. (2000). Development of language by hand and its connections to language by ear, mouth, and eye. Topics in Language Disorders, 20(4), 65-84. https://doi.org/10.1097/00011363-200020040-00007

Berninger, V. W. \& Abbott, R. D. (2010). Listening comprehension, oral expression, reading comprehension, and written expression: Related yet unique language systems in grades 1, 3, 5, and 7. Journal of Educational Psychology, 102(3), 635-651. https://doi.org/10.1037/a0019319

Biber, D. (2010). What can a corpus tell us about registers and genres? En A. O’Keeffe y M. McCarthy (Eds.). The Routledge Handbook of Corpus Linguistics (pp. 241-254). Routledge.

Biber, D. \& Conrad, S. (2009). Register, Genre, Style. Cambridge, Inglaterra: Cambridge University Press.

Bonomo, H., Mamberti, J. \& Miller, J. (2010). Tolerancia crítica y ciudadanía activa. Nueva York, NY: International Debate Education Association.

Burton, L., Nunes, T. \& Evangelou, M. (2021). Do children use logic to spell logician? Implicit versus explicit teaching of morphological spelling rules. British Journal of Educational Psychology, e12414. https://doi.org/10.1111/bjep.12414

Caravolas, M., Lervåg, A., Mousikou, P., Efrim, C., Litavský, M., Onochie-Quintanilla, E., ... \& Hulme, C. (2012). Common patterns of prediction of literacy development in different alphabetic orthographies. Psychological Science, 23(6), 678-686. https://doi.org/10.1177/0956797611434536

Coirier, P. \& Golder, C. (1993). Writing argumentative text: A developmental study of the acquisition of supporting structures. European Journal of Psychology of Education, 8(2), 169-181. https://doi.org/10.1007/BF03173160

Davidson, A. (2019). Writing: the re-construction of language. Language Sciences, 72, 134-149. https://doi.org/10.1016/j.langsci.2018.09.004

Decreto 187/2015 d'ordenació dels ensenyaments de l'educació secundària obligatòria. (2015). Recuperado de https://dogc.gencat.cat/ca/document-del-dogc/?documentId=701354

Dickinson, D. K. \& Tabors, P. O. (2001). Beginning literacy with language: Young children learning at home and school. Baltimore, MD: Paul H. Brookes.

Dockrell, J. E. \& Connelly, V. (2015). The role of oral language in underpinning the text generation difficulties in children with specific language impairment. Journal of Research in Reading, 38(1), 18-34. https://doi.org/10.1111/j.1467-9817.2012.01550.x

Duhaylongsod, L. (2017). Classroom Debates in Middle School Social Studies: Moving from Personal Attacks to Evidence and Reasoning. Middle Grades Research, 11(2), 99-115.

Felton, M. \& Kuhn, D. (2001). The development of argumentative discourse skill. Discourse Processes, 32(2-3), 135-153. https://doi.org/10.1080/0163853X.2001.9651595

Gaskell, M. G. \& Marslen-Wilson, W. D. (1998). Mechanisms of phonological inference in speech perception. Journal of Experimental Psychology: Human Perception and Performance, 24(2), 380. https://doi.org/10.1037/0096-1523.24.2.380

Golder, C. \& Coirier, P. (1996). The production and recognition of typological argumentative text markers. Argumentation, 10(2), 271-282. https://doi.org/10.1007/BF00180729

Graham, S. \& Harris, K. R. (2009). Almost 30 years of writing research: Making sense of it all with The Wrath of Khan. Learning Disabilities Research \& Practice, 24(2), 58-68. https://doi.org/10.1111/j.1540-5826.2009.01277.x

Graham, S. \& Harris, K. (2018). An examination of the design principles underlying a self-regulated strategy development study. Journal of Writing Research, 10(2), 139-187. https://doi.org/10.17239/jowr-2018.10.02.02 
Graham, S., McKeown, D., Kiuhara, S. \& Harris, K. R. (2012). A meta-analysis of writing instruction for students in the elementary grades. Journal of Educational Psychology, 104(4), 879-896. https://doi.org/10.1037/a0029185

Graham, S. \& Perin, D. (2007). A meta-analysis of writing instruction for adolescent students. Journal of Educational Psychology, 99(3), 445-476. https://doi.org/10.1037/0022-0663.99.3.445

Griffin, T. M., Hemphill, L., Camp, L. \& Wolf, D. P. (2004). Oral Discourse in the Preschool Years and Later Literacy Skills. First Language, 24(2), 123-147. https://doi.org/10.1177/0142723704042369

Hulme, C., Zhou, L., Tong, X., Lervåg, A. \& Burgoyne, K. (2019). Learning to read in Chinese: Evidence for reciprocal relationships between word reading and oral language skills. Developmental Science, 22(1), e12745. https://doi.org/10.1111/desc.12745

Hyland, K. (2004). Disciplinary discourses: Social interactions in academic writing. Ann Arbor, MI: University of Michigan Press.

Jones, S. M., LaRusso, M., Kim, J., Kim H. Y., Robert Selman, Uccelli, P., Barnes, S. P., Donovan, S. \& Snow, C. (2019). Experimental Effects of Word Generation on Vocabulary, Academic Language, Perspective Taking, and Reading Comprehension in High-Poverty Schools. Journal of Research on Educational Effectiveness, 12(3), 448-483. https://doi.org/10.1080/19345747.2019.1615155

Juel, C., Griffith, P. L. \& Gough, P. B. (1986). Acquisition of literacy: A longitudinal study of children in first and second grade. Journal of Educational Psychology, 78(4), 243. https://doi.org/10.1037/0022-0663.78.4.243

Kemper, M. J., Verhoeven, L. \& Bosman, A. M. (2012). Implicit and explicit instruction of spelling rules. Learning and Individual Differences, 22(6), 639-649. https://doi.org/10.1016/j.lindif.2012.06.008

Kieffer, M. J. \& Lesaux, N. K. (2010). Morphing into adolescents: Active word learning for English language learners and their classmates in middle school. Journal of Adolescent \& Adult Literacy, 54(1), 47-56. https://doi.org/10.1598/JAAL.54.1.5

Kintsch, W. \& Van Dijk, T. A. (1978). Toward a model of text comprehension and production. Psychological Review, 85(5), 363-394. https://doi.org/10.1037/0033-295X.85.5.363

Kline, R. B. (2011). Principles and practice of structural equation modeling. Nueva York, NY: Guilford Press.

Kuhn, D. \& Crowell, A. (2011). Dialogic argumentation as a vehicle for developing young adolescents' thinking. Psychological Science, 22(4), 545-552. https://doi.org/10.1177/0956797611402512

Larraín, A., Freire, P. \& Olivos, T. (2014). Habilidades de argumentación escrita: Una propuesta de medición para estudiantes de quinto básico. Psicoperspectivas, 13(1),94-107. https://doi.org/10.5027/psicoperspectivas-Vol13-Issue1-fulltext-287

Levesque, K. C., Kieffer, M. J. \& Deacon, S. H. (2017). Morphological awareness and reading comprehension: Examining mediating factors. Journal of Experimental Child Psychology, 160, 1-20. https://doi.org/10.1016/j.jecp.2017.02.015

Limpo, T. \& Alves, R. A. (2013). Teaching planning or sentence-combining strategies: Effective SRSD interventions at different levels of written composition. Contemporary Educational Psychology, 38(4), 328-341. https://doi.org/10.1016/j.cedpsych.2013.07.004

Lin, A. R., Lawrence, J. F., Snow, C. E. \& Taylor, K. S. (2016). Assessing adolescents' communicative self-efficacy to discuss controversial issues: Findings from a randomized study of the Word Generation program. Theory \& Research in Social Education, 44(3), 316-343. https://doi.org/10.1080/00933104.2016.1203852

Martins, M. A. \& Silva, C. (2006). The impact of invented spelling on phonemic awareness. Learning and Instruction, 16(1), 41-56. https://doi.org/10.1016/j.learninstruc.2005.12.005

Malvern, D., Richards, B., Chipere, N. \& Durán, P. (2004). A New Measure of Inflectional Diversity and its Application to English and Spanish Data Sets. En D. Malvern, B. Richards, N. Chipere, \& P. Durán, Lexical Diversity and Language Development (pp. 110-117). Londres, Inglaterra: Palgrave Macmillan.

Meneses, A., Uccelli, P., Santelices, M. V., Ruiz, M., Acevedo, D. \& Figueroa, J. (2018). Academic language as a predictor of reading comprehension in monolingual Spanish-speaking readers: Evidence from Chilean early adolescents. Reading Research Quarterly, 53(2), 223-247. https://doi.org/10.1002/rrq.192

Nelson, N. (2010). Language and literacy: Infancy through adolescence. Boston, MA: Allyn \& Bacon.

Perelman, C. (1997). El imperio retórico, Retórica y Argumentación. Bogotá, Colombia: Norma. 
Perfetti, C. A., Beck, I., Bell, L. C. \& Hughes, C. (1987). Phonemic knowledge and learning to read are reciprocal: A longitudinal study of first grade children. Merrill-Palmer Quarterly, 33(3), 283-319. Recuperado de https://www.jstor.org/stable/23086537

Ravid, D. \& Berman, R. A. (2010). Developing Noun Phrase Complexity at School Age: A Text-Embedded CrossLinguistic Analysis. First Language, 30(1), 3-26. https://doi.org/10.1177/0142723709350531

Ravid, D. \& Tolchinsky, L. (2002). Developing linguistic literacy: A comprehensive model. Journal of Child Language, 29(2), 417-447. https://doi.org/10.1017/S0305000902005111

Real Decreto 1105/2014, de 26 de diciembre, por el que se establece el currículo básico de la Educación Secundaria Obligatoria y del Bachillerato. (2015). Recuperado de https://www.boe.es/buscar/pdf/2015/BOE-A-2015-37-consolidado.pdf

Reznitskaya, A., Anderson, R. \& Kuo, L. (2007). Teaching and learning argumentation. Elementary School Journal, 107(5), 449-472. https://doi.org/10.1086/518623

Salas, N. (2010). Developmental and discursive underpinnings in the expression of Spanish noun phrases. Tesina de máster no publicada. Departamento de Lingüística General, Universitat de Barcelona

Salas, N., \& Caravolas, M. (2019). Dimensionality of early writing in English and Spanish. Journal of Literacy Research, 51(3), 272-292. https://doi.org/10.1177\%2F1086296X19858146

Salas, N., Birello, M., \& Ribas, T. (2020). Effectiveness of an SRSD writing intervention for low-and high-SES children. Reading and Writing, 1-28. https://doi.org/10.1007/s11145-020-10103-8

Salas, N., Birello, M., \& Cros, A. (en prep.). Embedding explicit linguistic instruction in an SRSD writing intervention.

Scholtz, Z. Sadeck, M., Hodges, M., Lubben, F. \& Braund, M. (2006). Argumentation about data: learners' ability to think critically. En C. Malcom (Ed.), Proceedings of the 14th Annual Conference of the South African Association for Research in Mathematics, Science and Technology Education (pp. 658-664). Sudáfrica: SAAEMSTRE.

Strategic Education Research Partnership (SERP) (s.f.). WordGen Weekly [Software]. Recuperado de https://www.serpinstitute.org/wordgen-weekly/discussion_and_debate

Shanahan, T., MacArthur, C. A., Graham, S. \& Fitzgerald, J. (2006). Relations among oral language, reading, and writing development. En Ch. MacArthur, S. Graham, \& J. Fitzgerald (Eds.), Handbook of Writing Research (pp. 171-183). Nueva York, NY: The Guilford Press.

Snowling, M. J. \& Hulme, C. (2020). Annual Research Review: Reading disorders revisited-the critical importance of oral language. Journal of Child Psychology and Psychiatry, 62(5), 635-653. https://doi.org/10.1111/jcpp.13324

Spencer, T. D. \& Petersen, D. B. (2018). Bridging oral and written language: An oral narrative language intervention study with writing outcomes. Language, Speech, and Hearing Services in Schools, 49(3), 569-581. https://doi-org.are.uab.cat/10.1044/2018_LSHSS-17-0030

Taylor, N. A., Greenberg, D., Laures-Gore, J. \& Wise, J. C. (2011). Exploring the syntactic skills of struggling adult readers. Reading and Writing, 25(6), 1385-1402. https://doi.org/10.1007/s11145-011-9324-9

Telenius, M., Yli-Panula, E., Vesterinen, V. \& Vauras, M. (2020). Argumentation within upper secondary school student groups during virtual science learning: Quality and quantity of spoken argumentation. Education Sciences, 10(12), 1-19. https://doi.org/10.3390/educsci10120393

Tolchinsky, L. (2004). The nature and scope of later language development. En R. A. Berman (Ed.), Language Development across Childhood and Adolescence (pp. 233-248). Ámsterdam, Holanda: John Benjamins.

Treiman, R. (2017). Learning to spell words: Findings, theories, and issues. Scientific Studies of Reading, 21(4), 265-276. https://doi.org/10.1080/10888438.2017.1296449

Uccelli, P., Barr, C., Dobbs, C., Galloway, E., Meneses, A. \& Sánchez, E. (2015). Core academic language skills: An expanded operational construct and a novel instrument to chart school-relevant language proficiency in preadolescent and adolescent learners. Applied Psycholinguistics, 36(5), 1077-1109. https://doi.org/10.1017/S014271641400006X

Uccelli, P. \& Meneses, A. (2015). Habilidades de lenguaje académico y su asociación con la comprensión de lectura en la escuela primaria y media: un nuevo constructo operacional. Miríada Hispánica, 10, 179-206. Recuperado de http://www.miriadahispanica.com/revista/46bdee9011025a5f574e7933d9ba68983c038150.pdf

Vázquez, M.J. (2014). Metalenguaje en la NGLE (oralidad y escrituridad). Cuadernos AISPI (4), 91-108. https://doi.org/10.14672/4.2014.1035 
Venville, G. \& Dawson, V. M. (2010). The Impact of a Classroom Intervention on Grade 10 Students' Argumentation Skills, Informal Reasoning, and Conceptual Understanding of Science. Journal of Research in Science Teaching, 47(8), 952-977. https://doi.org/10.1002/tea.20358

Vernon, S. \& Ferreiro, E. (1999). Writing development: A neglected variable in the consideration of phonological awareness. Harvard Educational Review, 69(4), 395-416. https:/doi.org/10.17763/haer.69.4.p411667586738x0w

Wagner, R. K., Puranik, C. S., Foorman, B., Foster, E., Wilson, L. G., Tschinkel, E. \& Kantor, P. T. (2011). Modeling the development of written language. Reading and Writing, 24(2), 203-220. https:/doi.org/10.1007/s11145-010-9266-7

Wen, H. \& Coker Jr., D. L. (2020). The role of discourse knowledge in writing among firstgraders. Journal of Writing Research, 12(2), 453-484. https://doi.org/10.17239/jowr-2020.12.02.05 